\title{
A New Realization of the Whitened Matched Filter Incorporating Easy Symbol Synchronization for High-speed Transmission
}

\author{
Cheng-Kun Wang Lin-Shan Lee \\ Department of Electrical Engineering, Rm 512 \\ National Taiwan University \\ Taipei, 10764, Taiwan
}

\begin{abstract}
The whitened matched filter (WMF) is the optimum receiver filter for pulse amplitude modulation (PAM) and quadrature amplitude modulation (QAM) transmissions in the presence of intersymbol interference (ISI) and additive white Gaussian noise (AWGN). The conventional realization of the WMF requires sophisticated algorithms for timing recovery such that it is not suitable for high-speed applications. In this paper a new realization of the WMF is proposed, which incorporates very easy timing recovery for high-speed transmissions such as optical fiber communications. The proposed realization scheme uses a specially designed filter to equalize the ISI, such that only relatively simple timing recovery circuitry for ISI-free signals will be needed.
\end{abstract}

\section{Introduction}

Forney developed the whitened matched filter (WMF) [1] as the optimum receiver filter for pulse amplitude modulation (PAM) and quadrature amplitude modulation (QAM) transmissions in the presence of intersymbol interference (ISI) and additive white Gaussian noise (AWGN). Additional signal processing can be applied to the WMF output to complete the optimum receiver design, e.g., maximum-likelihood sequence estimation (MLSE), zero forcing (ZF), decision-feedback equalization (DFE), linear minimum mean-square-error estimation (MMSE), and mean-square decision feedback equalization (MSDFE) etc. However, such WMF is seldom used in practical communication systems. Presently, the WMF finds its way only in the low-speed voice-band modem and some medium-speed applications where the enormous amount of computation required in signal processing to realize the WMF is both technically achievable and economically feasible, and is not adopted when the complexity is considered too expensive, or when the speed is simply too high such as in optical fiber communications.

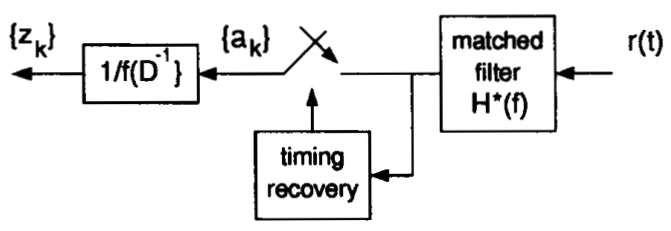

Figure 1: The whitened matched filter.

The WMF, as depicted in Figure 1, is composed of a matched filter, a symbol-rate sampler, and a transversal filter whose transfer function is obtained from the spectral factorization [1] of the pulse autocorrelation function. The primary difficulties in realizing the the WMF lie partly in the synthesis of the matched filter, and mainly in the timing recovery for the symbol-rate sampler. While equalization [2] can be used to synthesize the matched filter with ease, the complicated timing recovery for ISI-corrupted signals $[3,4,5]$ remains to be the major obstacle of the adoption of WMF in highspeed applications. In this paper a new realization of the WMF is proposed, which requires only relatively simple timing recovery circuits for ISI-free signals, therefore is especially suitable for high-speed applications. In the following, some background of the WMF will be briefly reviewed first for the development of this paper.

\section{A Brief Review of the WMF}

\subsection{The WMF}

Since QAM is basically composed of two PAMs in phase quadrature, it is sufficient to describe only the PAM. The block diagram of a baseband-equivalent PAM transmission system is depicted in Figure 2. Let $\left\{x_{k}\right\}$ be a sequence of integer symbols with finite length $N$ such

333.2.1 
that $0 \leq x_{k} \leq m-1,0 \leq k \leq N-1$. The impulse response and transfer function of the transmitting filter are $h(t)$ and $H(f)$, respectively. Then the transmitted signal $s(t)$ is

$$
s(t)=\sum_{k=0}^{N-1} x_{k} h(t-k T),
$$

where $T$ is the symbol duration, and the received signal $r(t)$ is the additive-noise corrupted version of $s(t)$

$$
r(t)=s(t)+n(t)
$$

where $n(t)$ is assumed to be a white Gaussian noise with two-sided power spectral density (PSD) $N_{0} / 2$. Let $D$ denotes the delay operator such that $D^{k} h(t)=h(t-$ $k T)$, then the symbol sequence $\left\{x_{k}\right\}$ can be expressed as $x(D)=\sum_{k=0}^{N-1} x_{k} D^{k}$, and the transmitted signal as

$$
s(t)=x(D) h(t) .
$$

At the receiver side a matched filter $H^{*}(f)$ followed by a symbol-rate sampler $[7,8]$ provides a set of sample values sufficient for estimating $x(D)$.

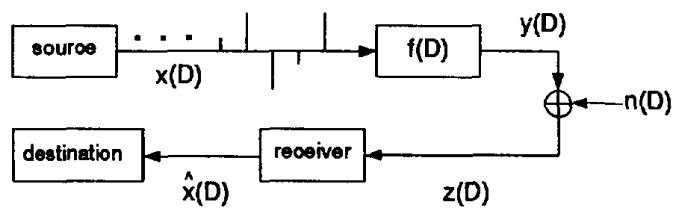

Figure 2: Baseband equivalent model of a PAM transmission system

Let $h(t)$ be a finite impulse response of length $L$ symbol durations, where $L$ is an integer. The autocorrelation coefficients of $h(t)$ are

$R_{k-k^{\prime}} \triangleq \begin{cases}\int_{-\infty}^{\infty} h(t-k T) h\left(t-k^{\prime} T\right) d t, & \left|k-k^{\prime}\right| \leq L-1 \\ 0 & \left|k-k^{\prime}\right| \geq L,\end{cases}$

and the pulse autocorrelation function of $h(t)$ is defined as

$$
R_{h h}(D) \triangleq \sum_{k=-\nu}^{\nu} R_{k} D^{k},
$$

where $\nu=L-1$ is called the span of $h(t)$. The symbolrate samples of the matched filter output are

$$
a_{k} \triangleq \int_{-\infty}^{\infty} r(t) h(t-k T) d t, \quad 0 \leq k \leq N-1,
$$

and the sequence $\left\{a_{k}\right\}$ can be expressed as

$$
a(D) \triangleq \sum_{k=0}^{N-1} a_{k} D^{k}
$$

Since

$$
\begin{aligned}
a_{k} & =\int_{-\infty}^{\infty} s(t) h(t-k T) d t+\int_{-\infty}^{\infty} n(t) h(t-k T) d t \\
& =\sum_{k^{\prime}} x_{k^{\prime}} R_{k-k^{\prime}}+n_{k}^{\mathrm{c}}
\end{aligned}
$$

where $n_{k}^{\mathrm{c}}$ is a noise sample, we have

$$
a(D)=x(D) R_{h h}(D)+n^{\mathrm{c}}(D),
$$

where $n^{c}(D)$ is a zero-mean colored Gaussian noise sequence with autocorrelation function $\frac{N_{0}}{2} R_{h h}(D)$. The pulse autocorrelation function $R_{h h}(D)$ is real and symmetric such that there exists the canonical factorization $R_{h h}(D)=f(D) f\left(D^{-1}\right)$ [1] where $f(D)$ is a real polynomial of degree $\nu$ that contains all the roots of $R_{h h}(D)$ outside the unit circle, and Equation (9) can be expressed as

$$
a(D)=x(D) f(D) f\left(D^{-1}\right)+n(D) f\left(D^{-1}\right),
$$

where $n(D)$ is a zero-mean white Gaussian noise sequence with variance $N_{0} / 2$. Hence a transversal filter characterized by $1 / f\left(D^{-1}\right)$ can be used to obtain the output sequence

$$
z(D)=a(D) / f\left(D^{-1}\right)=x(D) f(D)+n(D)
$$

in which the noise is white. Thus the WMF is the cascade of the matched filter, a symbol-rate sampler with correct sampling instants, and a transversal filter characterized by $1 / f\left(D^{-1}\right)$, as depicted in Figure 1 .

\subsection{Conventional Realization}

It is practically very difficult to realize the WMF directly in the form as shown in Figure 1 because the correct sampling instants at the matched filter output is very difficult to determine. In fact almost no realiztion of the WMF using this approach can be found. Instead, because of linearity, the transversal filter and the symbolrate sampler can be interchanged, and the matched filter and the transversal filter can be combined into one filter whose frequency response is $W(f)=H^{*}(f) / F_{-1}(f)$ where $F_{-1}(f)$ is the Fourier transformof $f\left(D^{-1}\right)$ and is periodic with period $1 / T$. This filter $W(f)$, also proposed by Forney [1] and in fact a direct consequence of the work of Ericson [6], can be view as the alternative form of the WMF. This form has the important interpretation that the receiving filter, usually realized by an equalizer, designed with the desired impulse response (DIR) set to $f(D)$, is a WMF [2]. Timing recovery can then be applied to this equalized output, which 
is now simpler than that in the original form in Figure 1 because the ISI is now less severe and the noise is white, as was demonstrated by several standard algorithms $[3,4,5]$. This realization scheme is plotted in Figure 3.

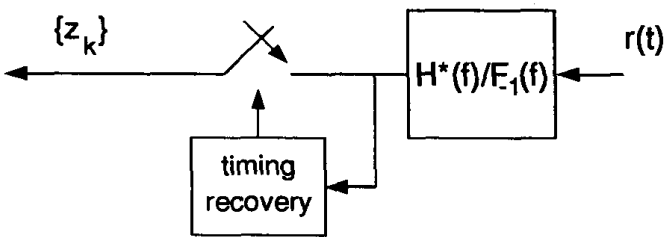

Figure 3: Alternative realization of WMF.

However, the ISI of the equalized pulse characterized by $f(D)$ can still be very severe such that all the algorithms $[3,4,5]$ for timing recovery requires enormous amount of computation in signal processing. This is the major obstacle of applying WMF in high-speed transmissions, such as optical fiber communications.

\section{The Proposed Realization Scheme}

A new realization of the WMF featured by easy timing recovery is proposed here as follows. As depicted in Figure 4, the proposed scheme consists of a matched filter in cascade with a filter $C(f)$ to be specified later, a symbol-rate sampler employing only simple ISI-free timing recovery circuitry, and a transversal filter characterized by $f(D)$. The filter $C(f)$ must satisfy two constraints:

1. It must be information-lossless after symbol-rate sampling.

2. The pulse shape at the filter output must satisfy the Nyquist zero-ISI criterion for easy timing recovery.

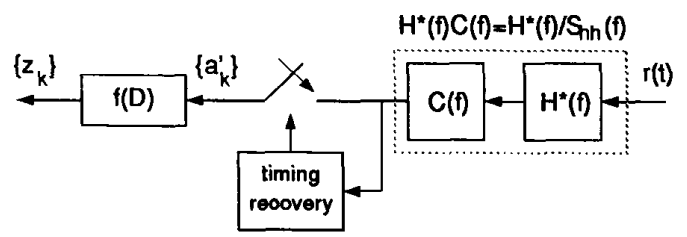

Figure 4: The proposed realization of the WMF.

By the theorem of reversibility [7] and the Nyquist sampling theorem, constraint 1 simply states that $C(f)$ is invertable and periodic with period $1 / T$. And by the Nyquist zero-ISI criterion, constraint 2 states that

$\sum_{n=-\infty}^{\infty} H(f-n / T) H^{*}(f-n / T) C(f-n / T)=$ constant.

Define the folded spectrum at the matched filter output as

$$
\begin{aligned}
S_{h h}(f) & \triangleq \sum_{n=-\infty}^{\infty} H(f-n / T) H^{*}(f-n / T) \\
& =\sum_{n=-\infty}^{\infty}|H(f-n / T)|^{2}
\end{aligned}
$$

which is the Fourier transform of the autocorrelation function $R_{h h}(D)$

$$
S_{h h}(f)=\left.R_{h h}(D)\right|_{D=e^{-j 2 \times T s} .}
$$

By the fact that $S_{h h}(f)$ and $C(f)$ are periodic with period $1 / T$, Equation (12) can be expressed as

$$
S_{h h}(f) C(f)=\text { constant, } 0 \leq f \leq 1 / T .
$$

Hence we require that $C(f)=1 / S_{h h}(f) \neq 0,0 \leq f \leq$ $1 / T$.

As depicted in Figure 4, the matched filter $H^{*}(f)$ and the filter $C(f)=1 / S_{h h}(f)$ can be combined into a single filter $H^{*}(f) / S_{h h}(f)$. The output of this filter is ISI-free for easy timing recovery, and the symbol-rate output are sent through a transversal filter characterized by $f(D)$ to produce the final output $z^{\prime}(D)$. We shall prove that this output is equivelent to that of the WMF output, that is, we shall show that

$$
z^{\prime}(D)=x(D) f(D)+n^{\prime}(D)
$$

where $n^{\prime}(D)$ should be a white Gaussian noise with variance $N_{0} / 2$. Below is the proof.

Because of the ISI-free receiver filter $H^{*}(f) / S_{h h}(f)$, the symbol-rate sampler output sequence is

$$
a^{\prime}(D)=\sum_{k=0}^{N-1} a_{k}^{\prime} D^{k}
$$

where

$$
a_{k}^{\prime}=x_{k}+n_{k}^{\text {co }}
$$

and $\left\{n_{k}^{\text {co }}\right\}$ is a colored Gaussian noise sequence whose PSD is

$$
\frac{N_{0}}{2} \sum_{n=-\infty}^{\infty}\left|H(f-n / T) / S_{h h}(f-n / T)\right|^{2}=N_{0} / 2 S_{h h}(f) \text {. }
$$

\subsection{3}


Hence we have

$$
a^{\prime}(D)=x(D)+n^{\mathrm{co}}(D)
$$

where the autocorrelation function of $n^{\mathrm{co}}(D)$ is $N_{0} / 2 R_{h h}(D)$ by the convolution theorem of the Fourier transform. The final output sequence is then

$$
\begin{aligned}
z^{\prime}(D) & =a^{\prime}(D) f(D) \\
& =x(D) f(D)+n^{c o}(D) f(D) \\
& =x(D) f(D)+n^{\prime}(D)
\end{aligned}
$$

where $n^{\prime}(D)$ is a Gaussian noise with autocorrelation function

$$
\begin{aligned}
\frac{N_{0}}{2 R_{h h}(D)} f(D) f\left(D^{-1}\right) & =\frac{N_{0}}{2 R_{h h}(D)} R_{h h}(D) \\
& =N_{0} / 2
\end{aligned}
$$

and is thus white with variance $N_{0} / 2$.

\section{Practical Considerations}

In low- and medium-speed applications where sophisticated digital signal processing can be used, the filter $H^{*}(f) / S_{h h}(f)$ can be implemented by a zero-forcing (ZF) fractionally spaced equalizer (FSE) [2]. In these situations the tap spacing $\tau$ must be chosen such that $1 / 2 \tau$ is larger than the cut-off frequency of the filter, i.e., $H^{*}(f) / S_{h h}(f)=0,|f| \geq 1 / 2 \tau$. The channels in these applications are usually slowly time-varying to justify the use of adaptive algorithms [2] to update the tap weights. The discrete transfer function $f(D)$ will then also vary slowly with time, and should be estimated from the tap weights.

In high-speed applications where digital signal processing is not feasible, such as in optical fiber communications, analog equalization can be used to realize $H^{*}(f) / S_{h h}(f)$ if both amplitude and phase equalizations are employed [9]. In analog equalizations, a ZF equalizer is usually expressed as

$$
H_{e q}(f)=\frac{\operatorname{R} \cos _{\beta}(f)}{H(f)}
$$

where $R \cos _{\beta}(f)$ is the raised cosine shaping filter with roll-off factor $\beta$. The roll-off factor is carefully chosen such that its cut-off frequency $(1+\beta) / 2 T$ is close to the the cut-off frequency of $H(f)$ to avoid excessive noise enhancement. Comparing $H_{e q}(f)$ with the proposed filter $H^{*}(f) / S_{h h}(f)$, its can be seen that we only have to replace the raised cosine filter $\operatorname{Rcos}_{\beta}(f)$ by $|H(f)|^{2} / S_{h h}(f)$, which is also an ISI-free filter. In these
ISI-free situations the optimal Data Transition Tracking Loop (DTTL) and Early-Late Gate Tracking Loop (ELGTL) [10] can be simply approximated by a phaselocked loop (PLL) $[11,12,13]$, which has already been implemented in giga-bit systems.

After the timing recovery, the transversal filter can be implemented by a analog-to-digital ( $A / D)$ converter and digital arithematic unit, which is not a problem even in giga-hertz range.

\section{Conclusion}

A new realization of the WMF incorporating easy symbol synchronization has been demonstrated in this paper. The proposed realization scheme is easy to implement, and is especially suitable for high-speed applications such as optical fiber communications.

\section{References}

[1] G. D. Forney, Jr., "Maximum-likelihood sequence estimation of digital sequence in the presence of intersymbol interference," IEEE Trans. Inform. Theory, vol. IT-18, pp. 363-378, May 1972.

[2] S. U. H. Qureshi, "Adaptive equalization," Proc. IEEE, vol. 73, pp. 1349-1387, Sept. 1985.

[3] R. D. Gitlin and J. salz, "Timing recovery in PAM systems," Bell Syst. Tech., vol. 50, pp. 1645-1669, May, 1971.

[4] S. U. H. Qureshi, "Timing recovery for equalized partial response systems," IEEE Trans. commun., vol. COM-24, pp. 1326-1330, Dec. 1976.

[5] L. E. Frank, "Carrier and bit synchronization in data communication," IEEE trans. commun., vol. COM-28, pp. 1107-1120, Aug. 1980.

[6] T. Ericson, "Structure of Optimum Receiving Filters in Data Transmission Systems," IEEE Trans. Inform. Theory, vol. IT-17, pp. 352-353, May 1971.

[7] J. M. Wozencraft and I. M. Jacobs, Principles of Communication Engineering. New York: Wiley, 1965.

[8] H. L. Van Trees, Detection, Estimation, and Modulation Theory, Part I. New York: Wiley, 1968.

[9] G. Cancellieri and F. Frosini, "Discusion on equalization in optical fiber transmission systems," $J$. Opt. Commun., vol. 3, pp. 146-151, 1982. 
[10] W. C. Lindsey and M. K. Simon, Telecommunication System Communication, Englewood Cliffs, N.J.: Prentice-Hall, 1973.

[11] F. M. Gardner, Phaselocked Techniques, New York: Wiley, 1979.

[12] C. R. Hogge, "Self correcting clock recovery circuit," IEEE J. Lightwave Tech., vol. LT-3, Dec. 1985.

[13] D. Shin, M. Park, and M. Lee, "Self-correcting clock recovery circuit with improved jitter performance," Electronic Letter, vol. 23, pp. 110-111, Jan. 1987. 